\title{
Near-UV Transmittance of Basalt Dust as an Analog of the Martian Regolith: Implications for Sensor Calibration and Astrobiology
}

\author{
G. M. Muñoz Caro *, E. Mateo-Martí and J. Martínez-Frías \\ Centro de Astrobiología, INTA-CSIC, Carretera de Ajalvir, km. 4, Torrejón de Ardoz, 28850 Madrid, \\ Spain

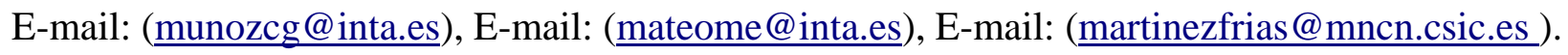

* Author to whom correspondence should be addressed.

Received: 17 February 2006 / Accepted: 22 June 2006 / Published: 27 June 2006

\begin{abstract}
The Martian regolith is exposed to solar irradiation in the near-UV (200-390 nm). Basalt is one of the main components of the dust on Mars surface. The near-UV irradiation of basalt dust on Mars is simulated experimentally in order to determine the transmittance as a function of the mass and thickness of the dust. This data can serve to quantify the absorption of dust deposited on sensors aiming to measure the UV intensity on Mars surface. The minimum thickness of the dust that corresponds to near-zero-transmittance in the near-UV is measured. Hypothetical Martian microorganisms living on the dusty regolith at deeper layers would be preserved from the damaging solar UV irradiation.
\end{abstract}

Keywords: Ultraviolet: solar system, Mars surface; Methods: laboratory, basalt; Instrumentation: photometers; Astrobiology

\section{Introduction}

The Martian surface is mainly composed of a dusty regolith and a rocky surface. It contains a mixture of highly oxidized secondary minerals and relatively pristine basalts [1]. Although recent works display evidence for igneous diversity and magmatic evolution on Mars [2], most Martian lava is basalt. Basalts are composed of plagioclase, feldspar, clinopyroxene, olivine, plus/minus sheet silicates and occur primarily in the equatorial to mid-latitude southern highlands regions [3]. Results from Mössbauer spectroscopy and X-ray fluorescence of dust particles captured from the Martian 
atmosphere by the magnets of the NASA's Mars Exploration Rovers show the presence of magnetite and olivine; this indicates a basaltic origin of the dust [4].

The ultraviolet (UV) flux on the surface of Mars is mainly in the near UV (200-390 nm) because of absorption and scattering in the Martian atmosphere. The integral UV flux on the Martian surface in the range 190-390 $\mathrm{nm}$ is estimated to be $\sim 210^{15}$ photons $\mathrm{cm}^{-2} \mathrm{~s}^{-1}$ [5].

A measurement of the UV transmittance using a $\sim 100 \mu$ m layer of JSC-1 Mars simulant (palagonite) placed onto a quartz cover slip observed a decrease of the UV flux of at least an order of magnitude [6]. The astrobiological implications of minerals on Mars surface were discussed in [7]. The UV absorbance of snow and ice, present in the polar ice caps, is lower than for dust and rocks. The penetration of UV-C radiation $(\lambda=190-280 \mathrm{~nm})$ using a $254 \mathrm{~nm}$ source in Antarctic ice and snow was measured, showing $40 \%$ reduction of the UV-C flux at a depth of $1 \mathrm{~cm}$ and about $90 \%$ reduction at a depth of $5 \mathrm{~cm}$ ([8] and ref. therein).

Basalt dust is one of the main components of the dusty regolith on Mars. The mean particle size of dust on the Martian surface is estimated to be a few $\mu \mathrm{m}$ [8]. In this work, the transmittance on the near-UV of basalt dust is investigated. The measurement of the transmittance of basalt dust, as a function of depth, serves to estimate the protection from UV irradiation due to the Mars regolith, which can prevent microorganisms from the damaging effects of the UV flux.

Dust will inevitably deposit on the instrumentation sent to the surface of Mars. Therefore, the data presented here could serve for the calibration of UV sensors in future Mars missions.

\section{Results and Discussion}

Let us first recall the basic concepts used for the interpretation of the results. The intensity of a beam of electromagnetic radiation with frequencies between $v$ and $v+d v$ is given by $I(v) d v$ where $I(v)$ is the spectral intensity. The total intensity is then given by

$I=\int_{0}^{\infty} I(v) d v$

If the beam crosses a slide in the $z$ direction, $I(v)$ decreases as

$\frac{d I(v)}{d z}=-\alpha(v) I(v)$

where $\alpha(v)$, the absorption coefficient, is characteristic of the material. Integration leads to LambertBeer's law:

$I(v)=I_{0}(v) e^{-\alpha(v) l}$

where $I_{0}(v)$ is the incident spectral intensity and $l$ is the length of the slide. Taking the neperian and 10logarithms we obtain 
$\log _{10} \frac{I_{0}(v)}{I(v)}=\frac{\alpha(v) l}{2.303}=A(v)$

where $A(v)$ is the absorbance of the slide. The transmittance $T(v)$ is defined as

$T(v)=\frac{I(v)}{I_{0}(v)}$.

The absorbance is therefore related to the transmittance by

$A(v)=-\log _{10} T(v)$.

For the case of an electromagnetic beam crossing an area that is only partially covered by absorbing material of constant thickness $l$, Eq. 5 becomes

$T(v)=\frac{I_{0}(v) e^{-\alpha(v) l} S_{d}+I_{0}(v)\left(S-S_{d}\right)}{I_{0}(v) S}=\left(e^{-\alpha(v) l}-1\right) \frac{S_{d}}{S}+1$,

where $S$ is the total area crossed by the beam and $S_{d}$ is the area covered by the absorbing material. This formula is an approach to the case of the $\mathrm{CaF}_{2}$ window partially covered by basalt dust, where $S$ corresponds to the total area of the $\mathrm{CaF}_{2}$ window, $S_{d}$ is the area of the $\mathrm{CaF}_{2}$ window covered by basalt dust, and it is assumed that the thickness $l$ of the dust layer over the area $S_{d}$ is constant, and zero where no dust is observed visually. The measurement of $S_{d}$ for each dust deposition was performed by taking a picture of the $\mathrm{CaF}_{2}$ window partially covered with dust, and integrating the area with the use of commercial software (analySIS 3.0 from Soft Imaging System). The thickness is obtained using the expression

$m=\rho V=\rho S \frac{S d}{S} l \rightarrow l=\frac{m}{\rho S \frac{S d}{S}}$

where $\rho=0.82 \mathrm{~g} \mathrm{~cm}^{-3}$ is the density that we obtained by weighting a cubic centimeter of the basalt dust. Fig. 1 shows the UV transmittance spectra corresponding to different mass depositions of basalt dust.

The total intensities, given by Eq. 1, in the range 200-400 nm (henceforth $I$ ), correspond to the areas under the irradiance spectra in Fig.1. The transmittance values, henceforth $T_{\text {measured }}$, were obtained using Eq. 3, where $I_{0}(v)$ was substituted by the $I$ value for $0.0 \mathrm{mg} \mathrm{cm}^{-2}$ and $I(v)$ by the value of $I$ for the different dust depositions. Inserting the values of $T_{\text {measured }}$ and $l$, the latter was obtained from Eq. 8, together with the $S_{d} / S$ ratio, we can calculate the value of $\alpha(v)$ in the 200-400 nm range. Finally, substituting the value of $\alpha(v)$, henceforth $\alpha_{d u s t}$, in Eq. 4 provides the values for the absorbance of the dust, $A_{\text {dust }}$, which in term can be expressed as the transmittance values, $T_{\text {dust }}$, using Eq. 6 . It is important to note that $A_{d u s t}$ and $T_{\text {dust }}$ correspond to the simple case where the $\mathrm{CaF}_{2}$ window is completely covered by basalt dust with a layer of constant thickness $l$, i.e. $S_{d}=S$ and

$T_{\text {dust }}=e^{-\alpha}$ dust $l$. 


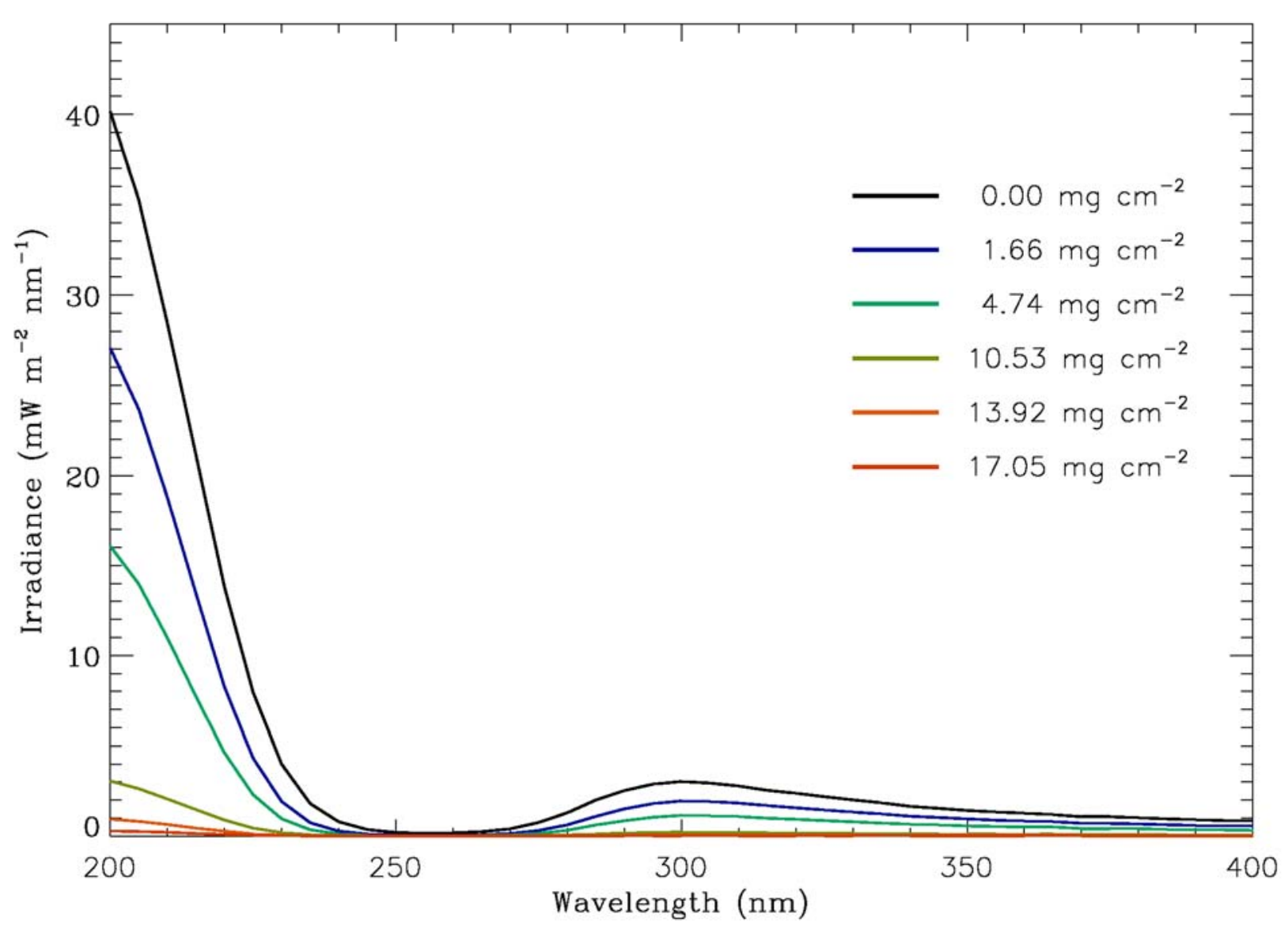

Figure 1. Transmittance spectra of deuterium lamp measured underneath sample for different basalt dust depositions with increasing mass.

The values of the experimental parameters for the range 200-400 nm, described above, are given in Table 1.

The absorption coefficient, $\alpha(v)$, should be constant for a given material at a given wavelength. The value $\alpha_{\text {dust }}=0.027 \mathrm{~mm}^{-1}$ is the most reliable, as it corresponds to depositions where the $\mathrm{CaF}_{2}$ window is almost fully covered by basalt dust $\left(S_{d} / S=0.95-0.99\right)$. The smaller $S_{d} / S$, the larger the errors introduced in the calculation of $\alpha_{\text {dust }}$, which might explain the large deviation of the $\alpha_{\text {dust }}$ value for the lowest dust density, $1.66 \mathrm{mg} \mathrm{cm}^{-2}$ and $S_{d} / S=0.41$. The lower value of $\alpha_{\text {dust }}$ for mass density $17.05 \mathrm{mg}$ $\mathrm{cm}^{-2}$ is due to the fact that the dust thickness corresponding to $T_{\text {dust }}=0.0$ was already reached for 13.92 $\mathrm{mg} \mathrm{cm}{ }^{-2}$ or $171 \mu \mathrm{m}$. The values of $T_{\text {measured }}$ correspond to the data points in Fig. 2 . The error bars result from the expression errorbar $=\sigma / \sqrt{(n)}$, where $\sigma$ is the standard deviation and $n=3$ the number of data points measured for each mass density.

The transmittance curve as a function of dust mass density (in $\mathrm{mg} \mathrm{cm}^{-2}$, red trace in Fig.2) was obtained for the case of a window fully covered by basalt dust of $5 \mu \mathrm{m}$ particle size, with uniform dust thickness $l$ (equivalent to uniform dust mass density), using Eq. 9 for $\alpha_{\text {dust }}=0.027 \mu \mathrm{m}^{-1}$. This value of $\alpha_{\text {dust }}$ corresponds to the near-UV range, $\lambda=200-400 \mathrm{~nm}$. However, Fig.1 shows that there is little 
dependence of the basalt dust transmittance with wavelength in the observed $\lambda=200-400 \mathrm{~nm}$ range, and therefore the value of $\alpha_{\text {dust }}$ is similar for a specific wavelength within that range.

Table 1. Experimental parameter values, see text for explanation.

\begin{tabular}{ccccccc}
\hline Mass density $\left(\mathrm{mg} \mathrm{cm}^{-2}\right)$ & $S_{d} / S$ & $l(\mu \mathrm{m})$ & $T_{\text {measured }}$ & $\alpha_{\text {dust }}$ & $A_{\text {dust }}$ & $T_{\text {dust }}$ \\
\hline 1.66 & 0.41 & 49 & 0.62 & 0.048 & 1.03 & 0.09 \\
4.74 & 0.72 & 80 & 0.36 & 0.027 & 0.95 & 0.11 \\
10.53 & 0.95 & 135 & 0.07 & 0.027 & 1.63 & 0.02 \\
13.92 & 0.99 & 171 & 0.02 & 0.027 & 1.99 & 0.01 \\
17.05 & 1.00 & 208 & 0.01 & 0.022 & 2.00 & 0.01 \\
\hline
\end{tabular}

In conclusion, as an application to UV sensor calibration, the effect of dust absorption deposited on UV sensors, aiming to measure the UV flux on the surface of Mars, can be accounted for using Eq.9 with $\alpha_{\text {dust }}=0.027 \mathrm{~m}^{-1}$ (red curve in Fig.2), for the case of a basalt dust layer with known uniform thickness or deposited mass density. For instance, it is found that a basalt dust layer of $\sim 1.1 \mu \mathrm{m}$ thickness reduces the transmittance from 100 to $97 \%$, that is, introduces an error of $3 \%$ on the determination of the UV flux on the surface of Mars. Transmittance drops to $1 \%$ at $170 \mu \mathrm{m}$ depth, and to $0.1 \%$ at $\sim 256 \mu \mathrm{m}$ depth. The flux at the surface of Mars at $250 \mathrm{~nm}$ is thought to be $\sim 3000$ times greater than that on Earth [8]. Such a reduction of the flux corresponds to a basalt dust thickness of $\sim 300 \mu \mathrm{m}$. Microorganisms living at such depths would be preserved from the damaging effects of UV irradiation on Mars surface. Future experiments will aim at providing the value of $\alpha_{\text {dust }}$ for other components of the Martian regolith.

For future missions aiming to measure the UV flux on the surface of Mars it is also relevant to have an estimation of the UV absorption on top of the UV detector due to dust deposition as a function of time. If the UV detector is positioned more than $20 \mathrm{~cm}$ above the surface, the sand and dust lifted from the surface by wind can be avoided [9]. According to this author, accumulation by settling of the dust suspended in the atmosphere occurs at a rate of $S_{d} / S=0.0036$ per day, i.e. $0.36 \%$ of the surface is covered in one day, for a cross-section weighted average particle radius of $r_{c}=2.75 \mu \mathrm{m}$ (he also indicates that in the best case, corresponding to the lowest dust deposition, the surface covered by dust is $0.03 \%$ per day, and in the worst case $2.9 \%$ per day). These values can be inserted in Eq. 7 to obtain the decrease in the transmittance as a function of time. For instance, assuming the worst case (after substitution of the values $S_{d} / S=0.029$ per day, $\alpha_{\text {dust }}=0.027 \mu \mathrm{m}^{-1}$, and $l=2 r_{c}=5.5 \mu \mathrm{m}$ in Eq. 7) the estimated transmittance value is $99.6 \%$ after one day; a dust layer fully covering the sensor with a $5.5 \mu \mathrm{m}$ average thickness would accumulate in 34.5 days, which corresponds to a transmittance value of $86 \%$, and evidences the need of a device to remove the dust periodically. Such a device is also required for the normal case where $86 \%$ transmittance (one dust layer) is attained after 278 days, but not for the best case since an $86 \%$ transmittance corresponds to 9 years. A dust deposition of $300 \mu \mathrm{m}$ thickness (55 dust layers) equivalent to near-zero- transmittance, would require about 5 years in the worst case scenario. However, the real case differs from a constant deposition rate: as observed by NASA's Mars Exploration Rovers wind events take place in the scale of months and were found to 
remove a large fraction of the deposited dust, while dust storms will deposit dust at higher rates than usual.

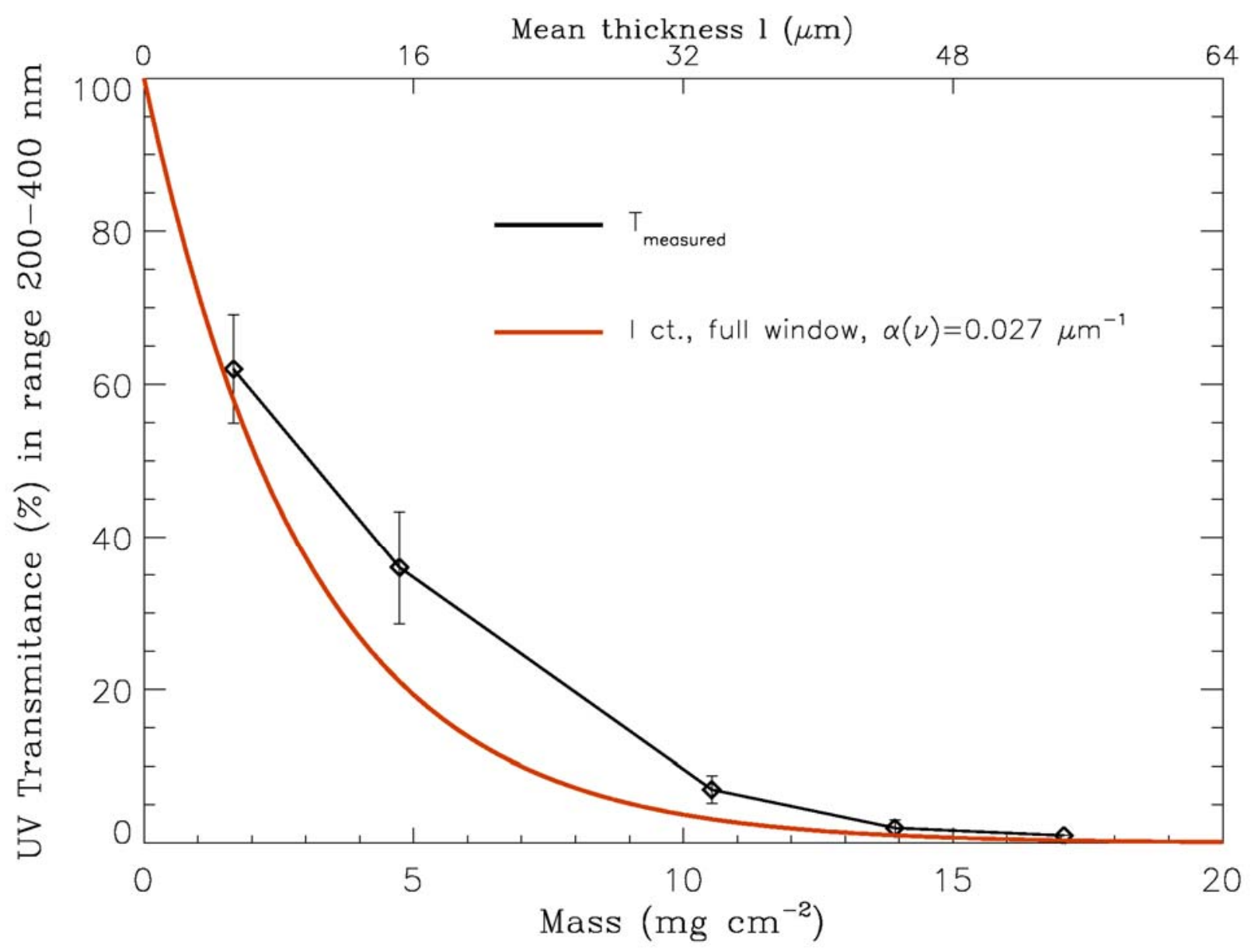

Figure 2.Transmitance of basalt dust for different mass depositions.

\section{Conclusions}

The reduction on UV transmittance as a function of the mass and thickness of basalt dust deposition was calibrated. It was found that the UV transmittance value practically drops to zero for a basalt dust thickness of $\sim 300 \mu \mathrm{m}$, and therefore microorganisms living at deeper layers would be protected from the damaging UV irradiation on Mars. The estimated surface covered by Martian dust in one day, due to settling of the dust suspended in the atmosphere, is $S_{d} / S=0.0036$ per day, i.e. $0.36 \%$ of the surface is covered in one day, for particles of $\sim 5.5 \mu \mathrm{m}$ diameter (upper and lower limits are $0.03 \%$ and $2.9 \%$ ), see [9]. These values can be inserted in Eq. 7 to obtain the decrease in the transmittance as a function of time in quiet periods, where no wind events or dust storms are taking place. Only in the most optimistic case, where $0.03 \%$ of the area is covered by dust in one day, dust settling would have little effect in the measure of UV flux on the surface of Mars for missions of a few months duration, but even in that case, occasional dust storms make necessary the use of a device for dust removal. 


\section{Experimental Section}

The experiments were performed in a planetary simulation chamber $[10,11]$, able to reproduce the atmospheric and surface temperature conditions of planetary objects. This experimental set-up, see Fig. 3 for a schematic view, makes feasible the in-situ irradiation and FTIR characterization of the sample under study. Our experiments have been carried out at room temperature under atmospheric pressure, due to the constraint to work with small dust size particles under low pressure conditions. This led to a decrease of the incident UV flux caused by ozone $\left(\mathrm{O}_{3}\right)$ absorption. Indeed, $\mathrm{O}_{3}$ is formed by UV irradiation of $\mathrm{O}_{2}$ for $\lambda<240 \mathrm{~nm}$. During photo-dissociation $\mathrm{O}_{3}$ absorbs UV light between 200 and $320 \mathrm{~nm}$ with maximum at $254 \mathrm{~nm}$ ([11], and ref. therein). $\mathrm{UV}$ absorption due to atmospheric $\mathrm{O}_{3}$ is also expected to occur on Mars.

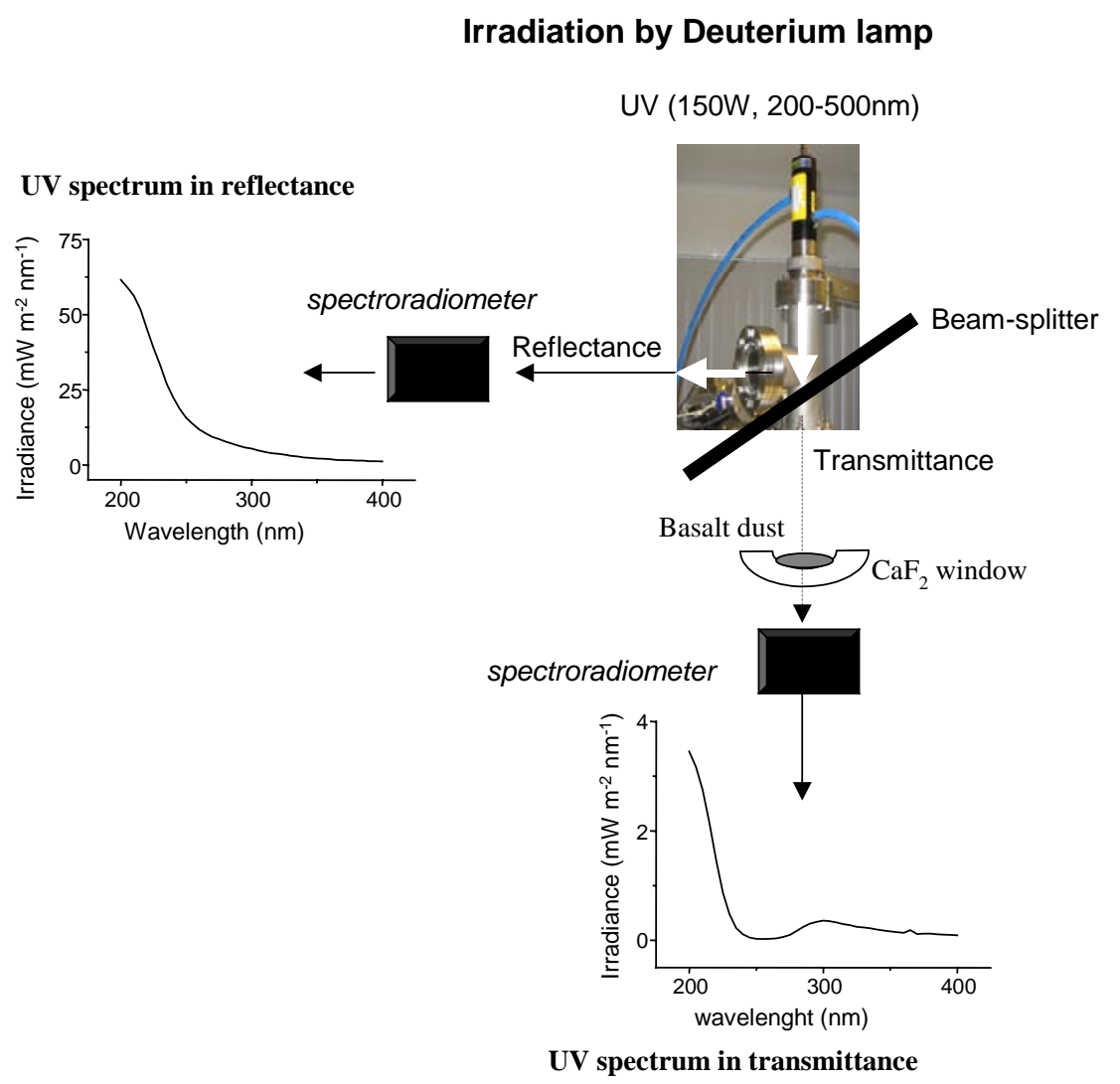

Figure 3. Scheme of the UV irradiation set-up on the planetary simulation chamber.

Basalt dust, with a mean particle size of $\sim 5 \mu \mathrm{m}$, was deposited on a near-UV transparent $\mathrm{CaF}_{2}$ window of $21 \mathrm{~mm}$ diameter, with the use of a sieve. From the geochemical point of view, ICP-MS analysis of the sample indicates that we used typical standard basalt. Irradiation was performed by means of a $150 \mathrm{~W}$ water-cooled deuterium UV lamp (Hamamatsu C3150), placed perpendicular and above the $\mathrm{CaF}_{2}$ window, with stable emission in the range 200-400 nm (6.2-3.1 eV). The irradiance spectrum of the deuterium lamp is a continuum that decreases for increasing photon wavelength (Fig.3, UV spectrum in reflectance); it was monitored during irradiation using a spectroradiometer (Bentham DMc150FC), placed underneath the $\mathrm{CaF}_{2}$ window. The UV flux measured at sample position, obtained by integration of the irradiance over the $200-400 \mathrm{~nm}$ wavelength range is $\mathrm{F}=2270 \mathrm{~mW} \mathrm{~m}{ }^{-2}$, which 
corresponds to $\mathrm{F}=2.310^{14}$ (6 eV photons) $\mathrm{cm}^{-2} \mathrm{~s}^{-1}$, about 10 times lower than the UV flux on the Martian surface (Sect.1).

\section{Acknowledgements}

We thank the Centro de Astrobiología (CAB) for finantial and institutional support, with special thanks to Prof. J. Pérez Mercader. We are thankful to J. A. Martín-Gago and O. Prieto-Ballesteros, who designed and constructed the planetary simulation chamber, and V. Souza-Egipsy for her assistance with the analySIS 3.0 software. We thank the REMS team members, in particular Prof. L. Vázquez, J. Gómez-Elvira, M. P. Zorzano and J. A. Rodríguez Manfredi. We acknowledge M. P. Martín Redondo and M. Fernández Sampedro for technical assistance. G.M.M.C. is supported by a Ramón y Cajal research program from the MCYT.

\section{References and Notes}

1. Bell III, J.F. Iron, sulfate, carbonate, and hydrated minerals on Mars. In Mineral spectroscopy: A tribute to Roger G. Burns, Geochimical Society Special Publications (M.D. Dyar, C. McCammon, and M.W. Schaefer, eds.) 1996, 359-380.

2. Christensen, P.R.; McSween, H.Y., Jr.; Bandfield, J.L.; Ruff, S.W.; Rogers, A.D.; Hamilton, V.E.; Gorelick, N.; Wyatt, M.B.; Jakosky, B.M.; Kieffer, H.H.; Malin, M.C.; Moersch, J.E. Evidence for magmatic evolution and diversity on Mars from infrared observations. Nature 2005, 436, 504-509.

3. Bandfield, J.L. Global mineral distribution on Mars. J. Geophys. Res. 2002, 107 (E6), 5042.

4. Goetz, W.; Bertelsen, P.; Binau, C.S.; Gunnlaugsson, H. P.; Hviid, S.F.; Kinch, K.M.; Madsen, D.E.; Madsen, M.B.; Olsen, M.; Gellert, R.; Klingelhöfer, G.; Ming, D.W.; Morris, R.V.; Rieder, R.; Rodionov, D.S.; de Souza, P.A.; Schröder, Ch.; Squyres, S.W.; Wdowiak, T.; Yen, A. Indication of drier periods on Mars from the chemistry and mineralogy of atmospheric dust. Nature 2005, 436 (7047), 62-65.

5. Mount, G.H.; Rottman, G.J. The solar absolute spectral irradiance 1150-3173-A - May 17, 1982. J. Geophys. Res. 1983, 88 (NC9), 5403-5410.

6. Pollack, J.B.; Colburn, D.S.; Flasar, F.M.; Kahn, R.; Carlston, C.E.; Pideck, D. Properties and effects of dust particles suspended in the Martian atmosphere. J. Geophys. Res. 1979, 84 (NB6), 2929-2945.

7. Martínez-Frías, J.; Amaral, G.; Vázquez, L. Astrobiological significance of minerals on Mars surface environment. Reviews in Environmental Science and Bio/technology 2006, in press.

8. Rontó, G.; Bérces, A.; Lammer, H.; Cockell, C.S.; Molina-Cuberos, G.J.; Patel, M.R.; Selsis, F. Solar UV irradiation conditions on the surface of Mars. Photochem. \& Photobio. 2003, 77 (1), 3440.

9. Landis, G.A. Dust obscuration of Mars solar arrays. Acta Astronautica 1996, 38, 885-891.

10. Martín Gago, J.A.; Mateo-Martí, E.; Prieto-Ballesteros, O.; Atienza, C.; Sobrado, J.M.; GómezElvira, J. PECAS: A new simulation chamber for studying planetary environments, LSPC 2005, poster presentation. 
11. Mateo-Martí, E.; Prieto-Ballesteros, O.; Sobrado, J.M.; Gómez-Elvira, J.; Martín Gago, J.A. A chamber for studying planetary environments and its application to astrobiology. Measurement Science and Technology 2006, 17, 1-7.

12. Bennett, C.J.; Kaiser, R.I. Laboratory studies on the formation of three $\mathrm{C}_{2} \mathrm{H}_{4} \mathrm{O}$ isomers acetaldehyde $\left(\mathrm{CH}_{3} \mathrm{CHO}\right)$, ethylene oxide $\left(\mathrm{c}-\mathrm{C}_{2} \mathrm{H}_{4} \mathrm{O}\right)$, and vinyl alcohol $\left(\mathrm{CH}_{2} \mathrm{CHOH}\right)$ - in interstellar and cometary ices. Astrophysical Journal 2005, 635, 1362-1369.

(c) 2006 by MDPI (http://www.mdpi.org). Reproduction is permitted for noncommercial purposes. 